\section{OC 8566 THE UNIVERSIDADE EDUARDO MONDLANE AND UNIVERSITY OF CALIFORNIA SAN DIEGO PARTNERSHIP, A PARADIGM FOR INSTITUTIONAL AND HUMAN RESOURCES CAPACITY BUILDING}

${ }^{1}$ Emilia Noormahomed*, ${ }^{2}$ Robert Scooley. 'Universidade Eduardo Mondlane, Maputo, Mozambique; ${ }^{2}$ University of California, San Diego, USA

10.1136/bmjgh-2019-EDC.33

Background Collaborations between lower- and middle-income countries (LMICs) and high-income countries (HICs) are often scientifically and structurally driven by the HICs. Here we aim to describe a paradigm shift in collaboration, exemplified by the collaboration between the Universidade Eduardo Mondlane and University of California, San Diego through the Medical Education Partnership Initiative (MEPI), in which the formulation of priorities and administrative infrastructure reside in the LMICs.

Methods We outline critical features of the MEPI partnership and compare with traditional models of collaboration, key features of success, lessons learned and the way forward.

Results LMIC programme partners translate broad programme goals and define metrics into priorities tailored to local conditions. Programme funds flow to a LMIC-based leadership group that contracts with HIC-based peers to provide technical and scientific advice and consultation in a reverse funds flow model. Emphasis is placed on strengthening administrative capacity within LMIC institutions and on creating communities of practice with common goals that resulted in expanded collaboration with European, Latin American, and African institutions. A rigorous monitoring and evaluation process modify programme priorities based on evolving opportunities to maximise programme impact.

Over five years, more than 63 research projects were designed, 19 of which received external funding and more than 40 manuscripts were published. Mozambican first-authored publications rose from $29 \%$ in $2001-2010 \%$ to $38 \%$ in 2011-2013.

Eighteen (18) residents completed internal medicine specialty training between 2010 and 2014. This represents a fourfold increase from over 1991 to 2000. Three (3) Master's programmes were created at Lurio University and 50 students successfully finished dissertations.

Conclusion Vesting LMIC partners with the responsibility for programme leadership and building administrative capacity in LMIC institutions substantially enhances programme relevance, impact and sustainability, and facilitates continuing acquisition of research and training funds to support professional development and institutional capacity building.

\section{OC 8568 A RANDOMISED CONTROLLED TRIAL OF ORAL IRON FOR TREATMENT OF POST-MALARIA ANAEMIA IN MALAWIAN CHILDREN COMPARING IMMEDIATE VERSUS DELAYED ADMINISTRATION}

${ }^{1}$ Kamija Phiri*, ${ }^{1}$ Herbert Longwe, ${ }^{1}$ Sarah White, ${ }^{1}$ Michael Esan, ${ }^{2}$ Feiko Ter Kuile, ${ }^{2}$ Bernard Brabin. 'College of Medicine, University of Malawi; Zomba, Malawi; 'Liverpool School of Tropical Medicine, UK

\subsection{6/bmjgh-2019-EDC.34}

Background Although universal provision of iron supplements to children is recommended by the WHO, it is not yet clear whether the administration of the supplements poses a risk in children in malaria-endemic areas. We investigate the effects of iron supplementation in children with post-malaria anaemia and haematological response with immediate and delayed (2 weeks) iron administration.

Methods A randomised double-blind clinical trial was conducted in Zomba and Blantyre between 2009 and 2013. All children aged 4 to 36 months with uncomplicated malaria and with iron deficiency were enrolled into the study. Malaria treatment was administered to all the children and they were randomly assigned to 3 groups as follows: immediate iron administration, delayed iron administration, or placebo. The children were followed up for 10 weeks, with their haematological recovery indices and adverse effects being monitored at 2, 4, 8 and 10 weeks. The primary outcome of the study was the proportion of children without anaemia (defined as $\mathrm{Hb}>10.9 \mathrm{~g} / \mathrm{dl}$ ) at the end of the iron supplementation period.

Results A total of 538 participants were randomised to immediate iron administration $(\mathrm{n}=183)$, delayed iron administration $(n=183)$, or placebo $(n=172)$. The incidence rate ratio (IRR) of being non-anaemic at the end of the follow-up period (10 weeks post-malaria infection) was 1.51 (95\% CI 1.17-1.94, $\mathrm{p}<0.001$ ) among immediate group versus the placebo group. There was no significant difference between delayed and placebo group (IRR 1.18, 95\% CI 0.91-1.55). Secondary analysis of risk of malaria and bacterial infection and iron markers at the end of the intervention period is underway and shall be presented at the conference.

Conclusion The results so far support the administration of iron immediately after completing antimalarial treatment in anaemic children, however safety results will be needed to be reviewed before conclusive recommendations.

\section{OC 8582 A PHASE IA/B STUDY TO ASSESS SAFETY AND IMMUNOGENICITY OF PLACENTAL MALARIA VACCINE CANDIDATE: PRELIMINARY RESULTS OF THE PRIMALVAC TRIAL}

${ }^{1}$ Amadou Konate, ${ }^{2}$ Laura Richert ${ }^{3}$ Arnaud Chêne, ${ }^{3}$ Jean-Philippe Semblat, ${ }^{4}$ Gwenaelle Roguet, ${ }^{5}$ Nadine Benhamouda, ${ }^{6}$ Mathilde Bahuaud, ${ }^{7}$ Nicolas Havelange, ${ }^{8}$ Alexis Kuppers, ${ }^{2}$ Cécilia Campion, ${ }^{2}$ Valérie Boilet, ${ }^{8}$ Sonia Gueguen, ${ }^{4}$ Pierre Loulergue, ${ }^{7}$ Odile Leroy, ${ }^{6}$ Frederic Batteux, ${ }^{5}$ Eric Tartour, ${ }^{7}$ Nicola $\mathrm{K}$ Viebig, ${ }^{2}$ Rodolphe Thiebaut, ${ }^{1}$ Sodiomon B Sirima, ${ }^{4}$ Odile Launay, ${ }^{3}$ Benoit Gamain*. ' Centre National de Recherche et de Formation sur le Paludisme, Burkina Faso; ${ }^{2}$ EUCLID/F-CRIN, CHU Bordeaux, INSERM, Bordeaux Population Health Research Center, UMR1219, France; ${ }^{3}$ INSERM U1134, Université Paris Diderot Sorbonne Paris-Cité, France; ${ }^{4}$ Assistance Publique Hôpitaux de Paris, CIC Cochin-Pasteur, France; ${ }^{5}$ INSERM U970, Université Paris Descartes Sorbonne Paris-Cité, Hôpital Européen Georges Pompidou, France; ${ }^{6}$ Université Paris Descartes, Sorbonne Paris Cité AP-HP, Département d'Immunologie Biologique, Groupe Hospitalier Cochin Broca Hôtel-Dieu, France; ' ${ }^{7}$ uropean Vaccine Initiative, Universitäts Klinikum Heidelberg, Germany; ${ }^{8}$ INSERM Pôle de Recherche Clinique, France

\subsection{6/bmjgh-2019-EDC.35}

Background Adhesion of P. falciparum-infected erythrocytes (PEs) to placental chondroitin-4-sulfate (CSA) has been linked to severe placental malaria (PM) outcomes. Evidence strongly supports the VAR2CSA variant surface antigen mediating PEs CSA-binding phenotype as the leading candidate for a PM vaccine. This study was conducted to assess the safety and immunogenicity of 3 different dosages $(20 \mu \mathrm{g}, 50 \mu \mathrm{g}$ and $100 \mu \mathrm{g}$ ) of the recombinant VAR2CSA protein (PRIMVAC), formulated with Alhydrogel or GLA-SE administered at days 0,28 and 56 . 